\title{
0 tempo médio entre o nascimento e a primeira mamada: o ideal e o real ${ }^{1}$
}

\section{The average time between the birth and the first breast-feeding: the ideal and the real}

\author{
El tiempo medio entre el nacimiento y primer amamantamiento: el ideal y el verdadero
}

\author{
Suzana Cordeiro da Silva', Leila Rangel da Silva", Luciana Fillies Bueno Mathias"'I
}

\begin{abstract}
RESUMO
Em 1992 o Brasil adotou a Iniciativa Hospital Amigo da Criança (IHAC) como estratégia para aumentar os índices de aleitamento materno. Este estudo se delimita no $4^{\circ}$ Passo desse programa. Os objetivos foram determinar 0 tempo médio entre o nascimento e a primeira mamada e verificar a sua relação com o tipo de parto. Trata-se de um estudo descritivo com abordagem quantitativa realizado no Instituto Fernandes Figueira (IFF). Foram entrevistadas 50 mulheres internadas com seus bebês em alojamento conjunto no período de março a maio de 2007. Os dados foram analisados pelo programa Epi-Info 3.4 de 2007. O tempo médio entre o nascimento e a primeira mamada dos partos cesáreos foi $6: 29 \mathrm{~h}$, quanto ao parto vaginal, foi $1: 55 \mathrm{~h}$. Os resultados encontrados vão contra a recomendação da IHAC.
\end{abstract}

Palavras chave: Enfermagem; Aleitamento Materno; Recém-Nascido.

\begin{abstract}
In 1992 Brazil adopted the Baby Friendly Hospital as a strategy to increase the indices of breast-feeding. This study is about the $4^{\text {th }}$ Step of this Program. The objectives were: to determinate the average time between the birth and the first breast-feeding and verify its relation with the type of birth. This is a descriptive study with a quantitative focus developed in Instituto Fernandes Figueira (IFF). There were interviewed 50 women interned with her babies in joint lodging in the period of March to May of 2007. The data had been analyzed by Epi-Info version 3.42007 program. The average time between the birth and the first breast-feeding of the Caesarean births was $6: 29 \mathrm{~h}$, and of the vaginal birth, it was $1: 55 \mathrm{~h}$. The results is not like the the Baby Friendly Hospital recommendation.
\end{abstract}

Key words: Nursing; Breast feeding; Newborn.

\section{RESUMEN}

En 1992 el Brazil adoptó la Iniciativa Hospital Amistoso del Bebé como una estrategia para aumentar los índices del amamantamiento. Este estudio está acerca del cuarto paso de este programa. Los objetivos fueram: determinar el tiempo medio entre el nacimiento y el primer amamantamiento, y verifican su relación con el tipo del nacimiento. Esto es un estudio descriptivo con un foco cuantitativo desarrollado en Instituto Fernandes Figueira (IFF). Allí fueron entrevistados 50 mujeres junto de sus bebés en el período de marzo a mayo de 2007. Los datos fueram analizados por el programa Epi-Infor versión de 3.4 de 2007. El tiempo medio entre el nacimiento y el primer amamantamiento de los nacimientos de Cesárea fue 6:29h, y del nacimiento vaginal, fue $1: 55 \mathrm{~h}$. Los resultados no están como la recomendación de la Iniciativa.

Palabras clave: Enfermería; Lactancia Materna; Recién-Nacido.

\footnotetext{
${ }^{1}$ Artigo construído a partir da monografia apresentada à Universidade Federal do estado do Rio de Janeiro - UNIRIO no primeiro semestre de 2007.

' Acadêmica do $9^{\circ}$ Período de Graduação em Enfermagem da Escola de Enfermagem Alfredo Pinto UNIRIO. Email: susu.nit@gmail.com

"Enfermeira, Doutora em Enfermagem, Professora Adjunta do departamento de Enfermagem Materno Infantil da Escola de Enfermagem Alfredo Pinto - UNIRIO. Email: Irangel@rio.com.br

III Enfermeira, Mestre em Enfermagem, especialista em Enfermagem Obstétrica do Instituto Fernandes Figueira. Email: lufillies@iff.fiocruz.br
} 


\section{NTRODUÇÃO}

A amamentação é a melhor maneira de proporcionar 0 alimento ideal para 0 crescimento saudável e o desenvolvimento dos recém-nascidos e possui importantes implicações para a saúde da mãe e do bebê. Para a mãe, auxilia na involução uterina, protege contra anemia, câncer de mama e ovário dentre outros. Para o bebê, protege contra infecções, alergias, além de melhorar o desenvolvimento neuro-motor infantil em geral e contribuir para o fortalecimento do elo mãe e filho $^{(1)}$. O aleitamento materno exclusivo tem um impacto significativo na redução da morbimortalidade infantil ${ }^{(2-3)}$.

Embora seja um ato natural, a amamentação é também um comportamento aprendido. Assim, ações que norteiam a saúde da mulher são necessárias, tanto para as mães quanto para os profissionais de saúde, no sentido de encorajamento e apoio para manter as práticas apropriadas de amamentação ${ }^{(4)}$. No Brasil, a mobilização em função do resgate ao aleitamento materno exclusivo até o sexto mês de vida começou na década de 70, acompanhando o movimento mundial. Desde então, o Ministério da Saúde tem investido em políticas voltadas para a promoção, proteção e apoio ao aleitamento materno. Em 1990, foi criada pelo Fundo das Nações Unidas para a Infância (UNICEF), juntamente com a Organização Mundial da Saúde (OMS), a Iniciativa Hospital Amigo da Criança (IHAC) que propõe a mobilização de serviços obstétricos hospitalares para a adoção de "Dez Passos para - Sucesso do Aleitamento Materno"(5). Ela propõe a adoção de normas e rotinas adequadas, treinamento dos profissionais e posterior avaliação das maternidades que desejam se credenciar. O Brasil aderiu a IHAC dois anos depois como uma estratégia para aumentar os índices de aleitamento materno.

Este estudo se delimita no $4^{\circ}$ Passo desse Programa cuja recomendação é que em pelo menos $80 \%$ dos partos normais e em pelo menos $50 \%$ das cesarianas as mulheres sejam ajudadas a amamentar na 1a meia hora após o nascimento, de preferência na sala de parto ou no centro cirúrgico, enquanto mãe e recém nato estão alertas e interagindo de forma que o contato pele a pele propicie a primeira sucção de forma natural e espontânea dentro da primeira hora de vida.

Apesar das recomendações de organismos nacionais e internacionais, a prática do aleitamento materno, no Brasil, ainda sofre algumas influências nos aspectos biológicos, sociais e culturais. A amamentação, além de biologicamente determinada, é socioculturalmente condicionada, tratando-se, portanto, de um ato impregnado de ideologias e determinantes que resultam das condições concretas de vida(6). A utilização de alimentos industrializados parece estar sendo disseminada nas práticas alimentares de crianças, possivelmente pela praticidade mas, também, pela divulgação massiva da indústria de alimentos, com mensagens veiculadas pela mídia e incorporadas como necessárias ${ }^{(7)}$.

O Brasil é um dos países da América Latina com menor prevalência de amamentação exclusiva e sua mediana é de apenas 23,4 dias $^{(8-9)}$. Estamos, portanto, muito distantes das recomendações da OMS e do Programa Nacional de aleitamento materno e nutrição do país que preconizam o aleitamento materno exclusivo por 6 meses. O desmame precoce é tido como conseqüência de inúmeros fatores associados aos aspectos psicossociais, sócio-culturais e físicos ${ }^{(6)}$.

Nesse momento anterior ao estabelecimento da amamentação, as mães necessitam de apoio e incentivo da equipe de saúde de um modo geral, e em especial, dos enfermeiros que estão presentes durante todo esse processo no alojamento conjunto ${ }^{(10)}$. Um tempo de espera longo até que a amamentação seja iniciada causa ansiedade para a mãe, estresse para o recém-nascido e pode comprometer o sucesso da amamentação. Tanto a falta de apoio na atenção primária à saúde, quanto práticas hospitalares inadequadas, como a separação mãe-filho no pós-parto imediato, o início tardio da primeira mamada e o uso inadequado de suplementos, contribuem para a redução do aleitamento materno exclusivo até os seis meses de idade $^{(11)}$.

A pesquisa "Gênero, Poder e Cidadania: a mulher é sujeito no processo decisório da amamentação ao nascimento quando o status de HIV é ignorado pelo serviço?" em fase de 
elaboração coordenada por De Oliveira(11) realizada em cinco Maternidades Amigas da Criança dentre elas o Instituto Fernandes Figueira, teve como objetivo geral analisar, a partir da vivência de mulheres com status de HIV ignorado pelo serviço por ocasião do parto, as questões de gênero, poder e cidadania, decorrentes das estratégias assistenciais adotadas pelos Hospitais Amigos da Criança do Sistema de Gestão de Alto Risco do Município do Rio de Janeiro em relação à ajuda ao início do aleitamento materno na primeira meia-hora após o nascimento. A partir dos resultados parciais da pesquisa foi possível perceber que a amamentação na primeira meia hora após o parto não vinha sendo prestada à maioria das mulheres com status de HIV ignorado pelo serviço. Desde então, surgiu o interesse em investigar se essa política vem sendo praticada entre as mulheres que possuíam exames negativos para HIV do pré-natal no momento do parto e que não tenham sofrido nenhuma intercorrência materna ou do neonato que pudesse adiar o início da amamentação.

A partir disso, levantaram-se as seguintes questões norteadoras: Qual é o tempo médio entre o nascimento e a primeira mamada? Qual é a relação entre este tempo médio e o tipo de parto?

Frente aos questionamentos traçou-se como objeto do estudo: $O$ tempo médio entre o nascimento e a primeira mamada. Para tanto, foram delimitados como objetivo desta investigação determinar o tempo médio do nascimento até a primeira mamada e verificar qual é a relação entre o tempo médio do nascimento até a primeira mamada entre puérperas que tiveram parto normal e parto cesáreo.

A justificativa deste estudo está na necessidade de se observar às práticas em relação à amamentação na realidade de um Hospital Amigo da Criança que é ainda, referência no Município para atendimento de casos com alto risco materno-fetal e Nacional de Banco de Leite Humano.

O Contato mãe-bebê e a amamentação na primeira hora de vida compõem o tema da Semana Mundial de Aleitamento Materno (SMAM) de 2007. "Amamentação na primeira hora, proteção sem demora" foi o slogan escolhido pela Aliança Mundial para Ação em Aleitamento Materno (WABA), o que sinaliza a relevância deste assunto.

\section{METODOLOGI A}

Trata-se de um estudo descritivo com abordagem quantitativa, pois este método permite descrever fatos e fenômenos relativos à realidade através da observação, descrição e categorização.

O cenário escolhido para o estudo foi o Instituto Fernandes Figueira (IFF) que constitui uma Unidade Técnico-Científica da Fundação Oswaldo Cruz (Fiocruz). Foi criado em 1924 com o nome de Abrigo Hospital Arthur Bernardes por iniciativa de Carlos Chagas e passou a chamar-se Instituto Fernandes Figueira em 1946. A partir daí, assumiu o papel de Centro Científico destinado a promover pesquisas relativas à higiene e à medicina da criança, estudos e pesquisas biomédicas sobre maternidade, infância, adolescência e problemas sociais correlatos ${ }^{(12)}$.

O IFF consiste em uma Unidade Hospitalar Amiga da Criança da Fundação Oswaldo Cruz, vinculada ao Sistema Único de Saúde (SUS) que desenvolve atividades de ensino, pesquisa e assistência.

A referida instituição atende à população materno-infantil e conta com ambulatórios de pediatria, pré-natal e ginecologia, além de unidades de internação como maternidade, unidade neonatal, pediatria, ginecologia e banco de leite humano, tendo como principal característica o atendimento a casos de alto risco materno - fetal.

O trabalho de campo ocorreu de 12 de março a 22 de maio de 2007. Inicialmente, a proposta para compor a população do estudo foi de 100 mulheres com teste HIV negativo, internadas em sistema de alojamento conjunto com seus bebês com Apgar no $5^{\circ}$ minuto acima de 7. Porém, devido ao grande número de mulheres excluídas por não atenderem aos critérios estabelecidos neste estudo, a amostra foi reduzida para 50 mulheres.

Foram analisados 217 prontuários, relatórios de partos do Centro Obstétrico, livros do laboratório de virologia e relatório de admissão da Unidade Neonatal do mês de 
março a maio de 2007 para coleta de dados secundários.

Foi realizado um estudo piloto para adequação do instrumento e validação das respostas através de comparações entre as entrevistas com as informações do prontuário. Percebeu-se que a categorização inicial para se investigar o tempo decorrido do nascimento à primeira mamada estava inadequada, porque levava a perda de dados significativos referentes ao intervalo de tempo para valores maiores de trinta minutos e menores ou iguais a uma hora. Para solucionar este problema optouse por questionar a hora exata da primeira mamada.

A seleção para inclusão das participantes no estudo foi feita em 3 momentos, primeiro através da análise dos prontuários a fim de selecionar as mães que não tinham feito teste rápido e bebês com Apgar maior que 7 no $5^{\circ}$ minuto. Depois no momento que antecedia a entrevista onde eram identificadas as mulheres internadas em regime de alojamento conjunto com seus bebês.

Por último, o momento após o período de coleta de dados, quando as informações das mulheres entrevistadas eram cruzadas com os dados do relatório do Centro Obstétrico, com os dados do livro do Laboratório de Virologia e com o relatório de admissão da Unidade Neonatal. Neste instante, foi descoberto que 7 mulheres entrevistadas constavam no livro de virologia como tendo feito o teste rápido, logo, foram excluídas e 7 novas entrevistas realizadas.

As entrevistas foram conduzidas utilizando-se um formulário semi-estruturado sobre dados reprodutivos maternos e sobre amamentação dos recém-nascidos durante o período de internação. Foram feitas mediante assinatura do Termo de Consentimento Livre e Esclarecido que encerra sobre confidencialidade das informações, anonimato, isenção de riscos e benefícios diretos e a não obrigatoriedade da participação no estudo, respeitando a Resolução № 196/96, do Conselho Nacional de Saúde ${ }^{(13)}$. O projeto foi submetido e aprovado pelo Comitê de Ética em Pesquisa do IFF, protocolo 0003.0.008.000-07 em 07 de fevereiro de 2007. Para armazenamento e análise dos dados foi utilizado o programa Epi-Info versão 3.4 de 2007.

\section{RESULTADOS E DI SCUSSÃO}

Durante o período da coleta de dados foram realizados 217 partos, 114 (52,5\%) foram cesarianas, $88(40,6 \%)$ vaginais e 15 $(6,9 \%)$ registros do Centro Obstétrico não continham o tipo de parto. Aproximadamente $57(26,3 \%)$ dessas mulheres foram submetidas ao teste rápido e $58(26,7 \%)$ tiveram bebês malformados. $15(6,9 \%)$ bebês nasceram mortos e $28(12,9 \%)$ tiveram outro tipo de intercorrência como prematuridade ou sofrimento fetal.

Gráfico 1: Distribuição dos nascimentos

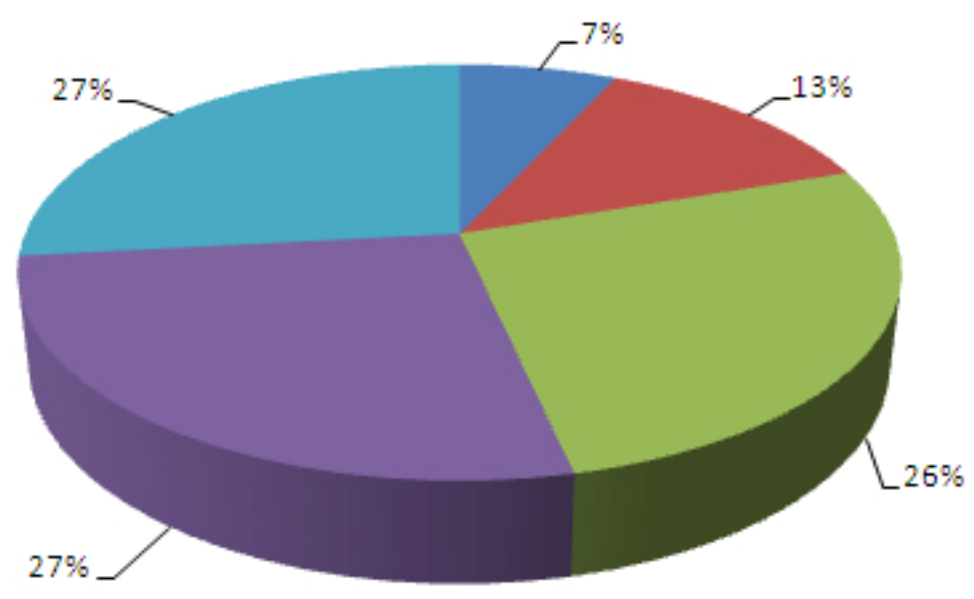

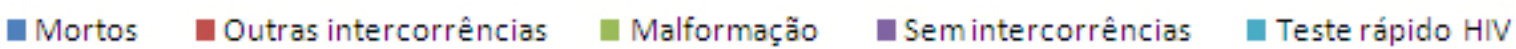


Foram entrevistadas 50 mulheres entre 15 e 43 anos, com idade média de 28,5 anos e mediana igual a 27 anos, sendo que $8(16 \%)$ eram adolescentes. Do total, 32 (64\%) mulheres estudaram entre 8 e 11 anos. Quase metade, $22(46 \%)$ possuía apenas um filho enquanto as demais possuíam 2 filhos em média. Dentre as que tiveram mais de um filho, a maioria, 25 (90\%) já havia amamentado. Quanto ao tipo de parto atual, 30 (60\%) tiveram cesarianas e $20(40 \%)$ tiveram partos vaginais.

Gráfico 2: Tipos de parto

- Parto normal Parto cesáreo

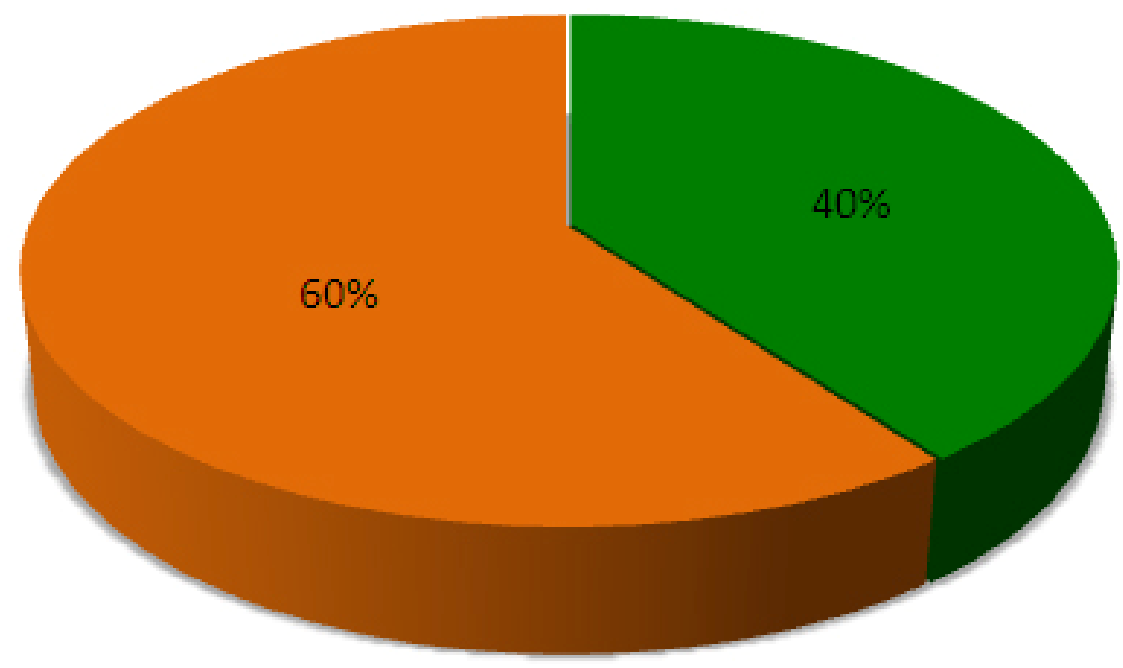

Pouco mais que a metade: $12(60 \%)$ das 20 mulheres que tiveram parto normal foram ajudadas a amamentar na sala de parto, e 12 $(60 \%)$ destas mulheres amamentaram seus bebês pela primeira vez nos primeiros 30 minutos.

No parto cesáreo essa oportunidade foi ainda menor, 6 (20\%) das 30 mulheres foram ajudadas a amamentar na sala de parto e 11 $(36,7 \%)$ destas amamentaram pela primeira vez entre 2 e 6 horas após o nascimento do seu bebê.

O intervalo de tempo para o início da amamentação variou de 5 a 22 horas e 34 minutos. A média desse tempo foi igual a 4 horas e 30 minutos. Quando estratificado por tipo de parto, o tempo médio entre o nascimento e a primeira mamada dos partos vaginais foi de 1 hora 55 minutos, nos cesáreos foi de 6 horas e 29 minutos, as medianas foram 36 minutos e 3 horas respectivamente. 
Gráfico 3: Tempo médio entre o nascimento e a primeira mamada

(tempo em horas)

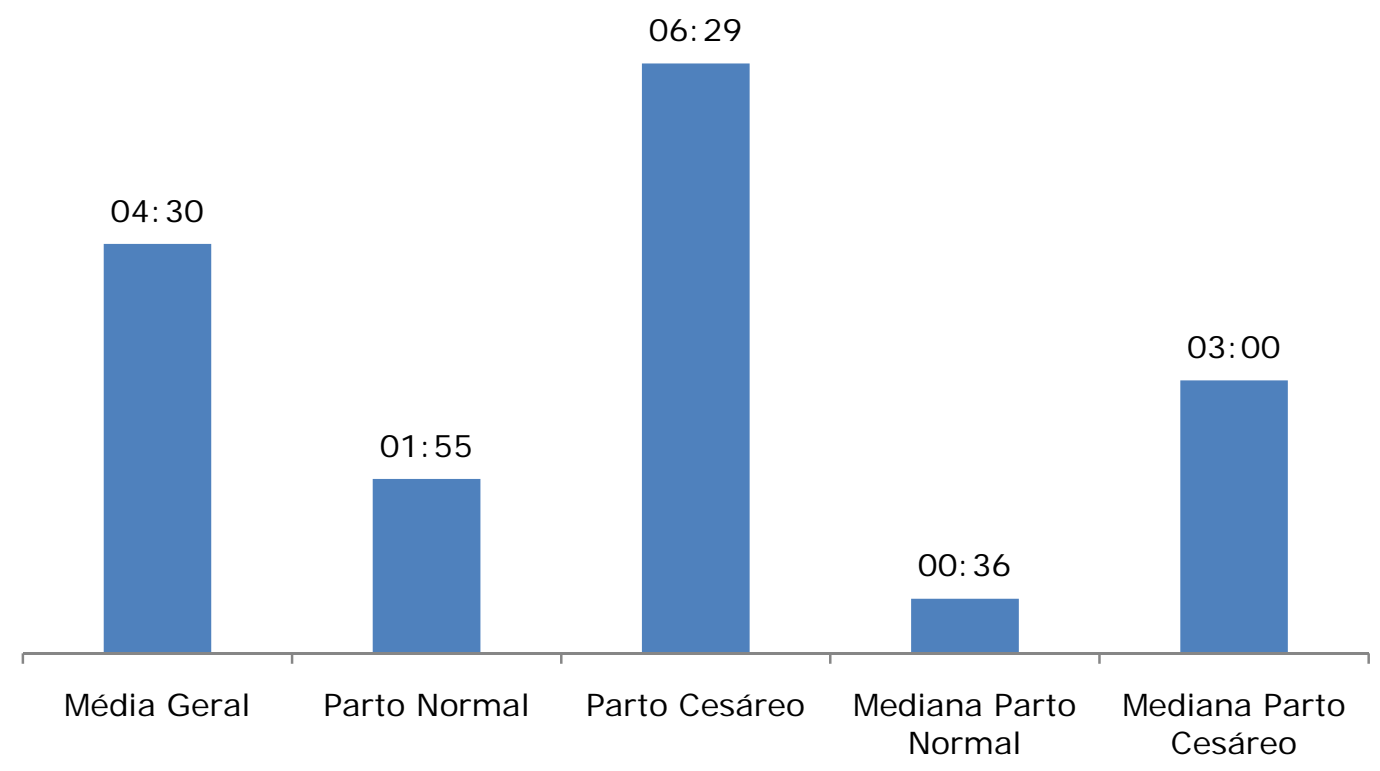

Dentre as 12 mulheres que tiveram parto normal e foram ajudadas a amamentar na sala de parto, $5(41,7 \%)$ foram ajudadas por enfermeiros, $4(33,3 \%)$ não lembram quem as ajudou, $1(8,3 \%)$ foi ajudada por obstetra e 2 $(16,7 \%)$ por pediatras.

Das 6 mulheres que tiveram cesariana e foram ajudadas a amamentar no centro obstétrico, 3 (50\%) foram ajudadas por enfermeiros, $2(33,3 \%)$ por pediatras e 1 $(16,7 \%)$ não lembra qual foi o profissional que a ajudou a amamentar.

É importante ressaltar que o fato do Instituto atender casos de risco fetal, onde muitas vezes se faz necessária a separação dos Recém-Nascidos de suas mães para unidades neonatais, dificultando assim o início precoce da amamentação, não influenciou no estudo, pois só foram incluídos mães e bebês sem intercorrência. Apesar das controvérsias sobre os anestésicos utilizados nos partos cesáreos e a amamentação, não há contra-indicação explícita de nenhum anestésico utilizado pelas mulheres que participaram do estudo à amamentação.

Enquanto os resultados encontrados sugerem que o incentivo a amamentação logo após o nascimento foi pouco praticado, no estudo feito por Toma e Monteiro(14) em São Paulo, não foi encontrada a prática de colocar o recém-nascido junto à mãe logo após o nascimento e permitir a amamentação na sala de parto ( $4^{\circ}$ Passo) em nenhum dos hospitais públicos e privados estudados.

Os resultados deste estudo não atendem a recomendação da IHAC em relação ao $4^{\circ}$ Passo. Poucas mulheres foram ajudadas a amamentar na sala de parto e nos primeiros 30 minutos. Esse valor variou quanto ao tipo de parto. Mulheres que tiveram parto normal foram mais ajudadas a amamentar na sala de parto e começaram a amamentar mais cedo que as que tiveram cesariana.

$O$ alto quantitativo dos partos tipo cesariana foi um fator prejudicial ao início precoce da amamentação neste estudo. Os índices de parto cesáreos $(60 \%)$ assim como os encontrados em um estudo realizado em Maringá - PR (45,3\%) encontram-se acima do preconizado pela Organização Mundial de Saúde, mesmo que o hospital seja referência para gestação de alto risco ${ }^{(14-15)}$.

Observamos que o papel da equipe de saúde para o início precoce da amamentação é muito importante, e neste estudo ficou nítido que essa também é uma responsabilidade da enfermagem. Assim como no estudo realizado no Recife, os achados mostram a importância da relação entre a educação, a saúde e o desenvolvimento social ${ }^{(16)}$. 


\section{CONCLUSÃO}

É importante salientar que o cumprimento do $4^{\circ}$ Passo da IHAC deve objetivar maior auxílio às mães e aumentar os índices de aleitamento materno, além de ser necessário para a manutenção do título Hospital Amigo da Criança.

O tempo médio entre o nascimento e a primeira mamada ainda é muito alto, é preciso valorizar a oportunidade do momento de alerta da mãe e bebê para reduzir a demora entre o nascimento e a primeira mamada, principalmente nas cesarianas.

É preciso manter o esforço a fim de reduzir o quantitativo das cesarianas quando possível.

Finalmente, para que este problema seja solucionado não basta a Instituição proporcionar cursos de capacitação, é preciso que os profissionais de saúde sintam-se coresponsáveis quanto a causa da amamentação, pois assim, certamente estaremos ajudando a mulher, a criança, a família, a sociedade e quiçá o planeta.

\section{REFERÊNCI AS}

1. Rea MF. Os benefícios da amamentação para a saúde da mulher. Jornal de Pediatria. 2004;80(5): S142-6.

2. NOTE-2001-07/The optimal duration of exclusive breastfeeding [Internet]. Geneva: World Health Organization (SW) [update 2006 jul 10, cited 2008 jul 16]. The optimal duration of exclusive breastfeeding: results of a WHO systematic review. Available from: $\quad$ http://www.who.int/inf-pr2001/en/note2001-07.html

3. Marques RFSV, Lopez FA, Braga JAP. O crescimento das crianças alimentadas com leite materno exclusivo nos primeiros 6 meses de vida. J ornal de Pediatria. 2004; 80(2): 99-105.

4. Alves VH. O ato da amamentação: um valor em questão ou uma questão de valor? 2003 [tese]. [Rio de Janeiro]: Escola de Enfermagem Anna Nery/UFRJ; 2003.

5. Ministério da Saúde; Secretaria de Vigilância em Saúde, Secretaria de Atenção à Saúde. Manual normativo para profissionais de saúde de maternidades da Iniciativa Hospital Amigo da Criança - Referências para mulheres HIV positivas e outras que não podem amamentar. Brasília (Brasil): Ministério da Saúde; 2004.

6. Silva MBC, Moura MEB, Silva AO. Desmame precoce: representações sociais de mães. Revista Eletrônica de Enfermagem [Internet]. 2007 [cited 2008 jul 12]; 9(1):31-50. Available from: http://www.fen.ufg.br/revista/v9/n1/pdf/ v9n1a03.pdf.

7. Machado MMT, Galvão MTG, Kerr-Pontes LRS, Cunha AJLA, Leite AJM, Lindsay AC, et al. Acesso e utilização de fórmula infantil e alimentos entre crianças nascidas de mulheres com HIV/AIDS. Revista Eletrônica de Enfermagem [Internet]. 2007 [cited 2008 jul 12] 9(3):699-711. Available from: http://www.fen.ufg.br/revista/v9/n3/pdf/ v9n3a10.pdf

8. Rea MF. Reflexões sobre a amamentação no Brasil: de como passamos a 10 meses de duração. Cadernos de Saúde Pública. 2003.(19 Sup 1):37-45.

9. Ministério da Saúde; Pesquisa de prevalência do aleitamento materno nas capitais e no Distrito Federal. Brasília (Brasil): Ministério da Saúde; 2001.

10. Ministério da Saúde. Programa de Humanização do Pré-natal e Nascimento. Portaria № 569 de 1o de julho de 2000. Brasília (Brasil): Ministério da Saúde; 2000.

11. Oliveira MIC, Camacho LAB. Impacto das unidades básicas de saúde sobre a prevalência de aleitamento materno exclusivo. Revista Brasileira de Epidemiologia. 2002; 5(1):41-51.

12. IFF - Instituto Fernandes Figueira [Internet]. Rio de Janeiro: Ministério da Saude (BR) [Internet] 2008 [update 2008 jun 27, cited 2008 jul 16]. Nossa história começa em 1924... Available

from: http://www. iff.fiocruz.br/textos/hist.htm 13. Ministério da Saúde; Comissão Nacional de Ética em Pesquisa, Conselho Nacional de Saúde. Cadernos Técnicos - Normas para pesquisas envolvendo seres humanos. Brasília (Brasil): 2000.

14. Toma TS, Monteiro CA. Avaliação da promoção do aleitamento materno nas maternidades públicas e privadas do Município de São Paulo. Revista de Saúde Pública. 2001; 35(5):409-14.

15. Knupp VMAO, Melo ECP, Oliveira RB. Distribuição do parto vaginal e da cesariana no 
município do Rio de Janeiro no período de 2001 a 2004. Escola Anna Nery Revista de Enfermagem. 2008; 12(1):39-44.

16. Campana HCR, SMP. Levantamento dos partos cesárea realizados em um hospital universitário. Revista Eletrônica de Enfermagem [Internet] 2007 [cited 2008 jul 16] 9(1): 51-63. Available from: http://www.fen.ufg.br/revista/v9/n1/pdf/ v9n1a04.pdf

17. Ministério da Saúde. Parto aborto e puerpério: assistência humanizada à mulher. Brasília (Brasil): Ministério da Saúde; 2001.

18. Morimura MCR, Mendes MDC, Souza Al et al. Freqüência de testagem rápida para o HIV durante a admissão para o parto em puérperas no Instituto Materno Infantil Prof. Fernando Figueira, IMIP. Revista Brasileira de Saúde Materno Infantil. 2006;6 Suppl. 1:69-76.

Artigo recebido em 08.10.07

Aprovado para publicação em 30.09.08 